\title{
Anatomical landmarks for transnasal endoscopic skull base surgery
}

\author{
Kishore Sandu $\cdot$ Philippe Monnier $\cdot$ Philippe Pasche
}

Received: 12 April 2011 / Accepted: 27 June 2011 / Published online: 9 July 2011

(C) Springer-Verlag 2011

\begin{abstract}
Resection of midline skull base lesions involve approaches needing extensive neurovascular manipulation. Transnasal endoscopic approach (TEA) is minimally invasive and ideal for certain selected lesions of the anterior skull base. A thorough knowledge of endonasal endoscopic anatomy is essential to be well versed with its surgical applications and this is possible only by dedicated cadaveric dissections. The goal in this study was to understand endoscopic anatomy of the orbital apex, petrous apex and the pterygopalatine fossa. Six cadaveric heads ( 3 injected and 3 non injected) and 12 sides, were dissected using a TEA outlining systematically, the steps of surgical dissection and the landmarks encountered. Dissection done by the "2 nostril, 4 hands" technique, allows better transnasal instrumentation with two surgeons working in unison with each other. The main surgical landmarks for the orbital apex are the carotid artery protuberance in the lateral sphenoid wall, optic nerve canal, lateral optico-carotid recess, optic strut and the V2 nerve. Orbital apex includes structures passing through the superior and inferior orbital fissure and the optic nerve canal. Vidian nerve canal and the V2 are important landmarks for the petrous apex. Identification of the sphenopalatine artery, V2 and foramen rotundum are important during dissection of the pterygopalatine fossa. In conclusion, the major potential advantage of TEA
\end{abstract}

Presented as an oral contribution at the Annual meeting of the Swiss Otorhinolaryngological Society, 2009 Geneva.

K. Sandu $(\varangle) \cdot$ P. Monnier $\cdot$ P. Pasche

Department of Otorhinolaryngology,

University Hospital CHUV, Lausanne, Switzerland

e-mail: Kishore.Sandu@chuv.ch to the skull base is that it provides a direct anatomical route to the lesion without traversing any major neurovascular structures, as against the open transcranial approaches which involve more neurovascular manipulation and brain retraction. Obviously, these approaches require close cooperation and collaboration between otorhinolaryngologists and neurosurgeons.

Keyword Transnasal endoscopic skull base anatomy . Anatomical landmarks · Endonasal $\cdot$ Skull base surgery

\section{Introduction}

Detailed knowledge of nasal and paranasal anatomy, including the anterior and middle skull base, is a fundamental requirement for surgical treatment of pathologies involving these anatomical areas. Cadaver dissection assisted by endoscopy is a valid approach for acquiring a true three-dimensional mental image rather than schematic anatomic knowledge. Also, otorhinolaryngologists are able to acquire appropriate practical skills that serve as basis for mastering these complex interventions. Disorientation of the surgical field is the major risk faced by a surgeon during endoscopy. An in-depth knowledge of endonasal anatomy improves the sense of depth to identify anatomic landmarks and sites at risk, and thus can avoid iatrogenic damage. Another crucial prerequisite for mastering the most complex surgical situations is knowledge of the neighbouring anatomical structures delimiting the sinonasal structures like the orbit, anterior and middle skull base.

The endoscopic transnasal transsphenoidal approach has now been adopted for treatment of several midline anterior skull base lesions $[1,2]$. It is versatile and minimally invasive as compared to open neurosurgical procedures. It offers 
a lower morbidity and mortality rate when compared to open transcranial procedures leading to shorter hospital stay. A variety of innovative skull base approaches (including anterior, anterolateral and posterolateral routes, which often require extensive neurovascular manipulation to gain access to the lesion) have been developed to allow resection of extra-axial lesions of the anterior skull base that are outside the sella, immediate parasellar area, or orbit but may be in close contact with the cavernous sinus or the carotid artery. In contrast to the traditional cranial base surgical approaches, the endonasal technique offers a direct approach without brain retraction offering the surgeon an ability to operate safely and effectively in a precarious surgical field [3, 4]. Furthermore, the constant improvements in diagnostic imaging techniques and the increasing use of image guidance systems during endoscopic endonasal procedures have provided increasing accuracy and safety for this approach, allowing improved, constant surgical orientation in an anatomically complex area.

We present an anatomical dissection study to define application of extended endoscopic endonasal approach to the midline skull base concentrating mainly on three areas-orbital apex, petrous apex and pterygopalatine fossa. The study was performed to understand better the complex anatomical relationships of the structures involved in the approach, and become more familiar with the endoscopic views and associated skills which are quite different from traditional microscopic approach. Surgery would mean collaboration between otorhinolaryngologists and neurosurgeons who need to be equally acquainted with the endonasal anatomy and endoscopic skills so as to excise the lesions correctly and manage possible complications.

\section{Materials and methods}

Transnasal endoscopic dissection was performed in six cadaveric heads (12 sides), of which three were noninjected and three heads had an intravascular injection of coloured liquid silicone using a previously described technique [1]. The heads were stored in $75 \%$ alcohol solution. Cadaveric dissections were performed and the specimen stored in the Anatomy laboratory of the University Hospital, Lausanne. We used Storz ${ }^{\mathrm{R}} 0^{\circ}, 30^{\circ}, 70^{\circ} 4 \mathrm{~mm}, 18$ and $30 \mathrm{~cm}$ rod lens rigid endoscopes fitted with an endoscope rinsing system (Karl Storz and Co., Tuttlingen, Germany). The endoscope was connected to a Xenon cold light source via fibreoptic cable and to a Stryker ${ }^{\mathrm{R}}$ video viewing and recording column to carefully document every surgical step and photograph important landmarks. The photographs were edited with Adobe $^{\mathrm{R}}$ Photoshop CS3 program. During edition, additional colouring of important bony landmarks and neurovascular structures was done for better identification of the surface anatomy. Routine Storz sinuscopic and pituitary instruments were used for dissection. Long neurosurgical high-speed cutting and diamond burrs were used for bone drilling. Cadaveric specimens were placed supine with the head in neutral position.

\section{Surgical dissection}

Nasal preparation and diagnostic nasal endoscopy

The dissection begins by cleaning of the nasal cavities with $\mathrm{Q}$ tips and cotton pledgets. The 1st diagnostic pass is along the floor of the nasal cavity between the inferior turbinate and the nasal septum up to the posterior choana. The middle turbinate is identified rostrally to the inferior turbinate. Eustachian tube opening with its tubal elevation is seen in the cavum. The 2 nd pass is made to identify the middle turbinate with its meatus. The $3 \mathrm{rd}$ pass is performed to visualise the natural sphenoid ostium passing the endoscope between the septum and middle turbinate. The ostium is positioned in the sphenoethmoidal recess, medial to the middle turbinate and behind the superior turbinate typically $1 \mathrm{~cm}$ above the roof of the posterior choana. All surgical steps are performed using a $0^{\circ}$ endoscope. The $30^{\circ}$ and $70^{\circ}$ scopes are used to see hidden areas needing angled visualisation.

\section{Preparation of surgical corridor}

The right middle turbinate is medialised and resected leaving a small stub attached to the skull base which can be used for future reference. Uncinate process is excised to visualise the hiatus semilunaris leading to the ethmoidal bulla. A complete anterior and posterior ethmoidectomy with excision of the basal lamella of the middle turbinate is performed. Lamina papyracea and the orbital periosteum, roof of the ethmoid and skull base with anterior ethmoid artery and the frontonasal recess are visualised (Fig. 1). Maxillary sinus ostium is identified with a curved suction tip following the superior edge of the inferior turbinate. The suction tip dips into the ostium at the junction of middle and posterior third of the inferior turbinate on the lateral nasal wall. Posterior ethmoid artery is identified on the skull base just anterior to the anterior sphenoid wall. A large posterior septectomy is done to allow a 2 nostril, 4 hands technique which in turn allows increased instrumentation and introduction of two or three instruments in the nasal cavity along with the endoscope at the same time. Anterior wall of the sphenoid sinus is excised bilaterally leaving rostrum of the sphenoid intact. Intersphenoidal septum and the entire sphenoid sinus mucosa is removed. Figure 2 shows the 


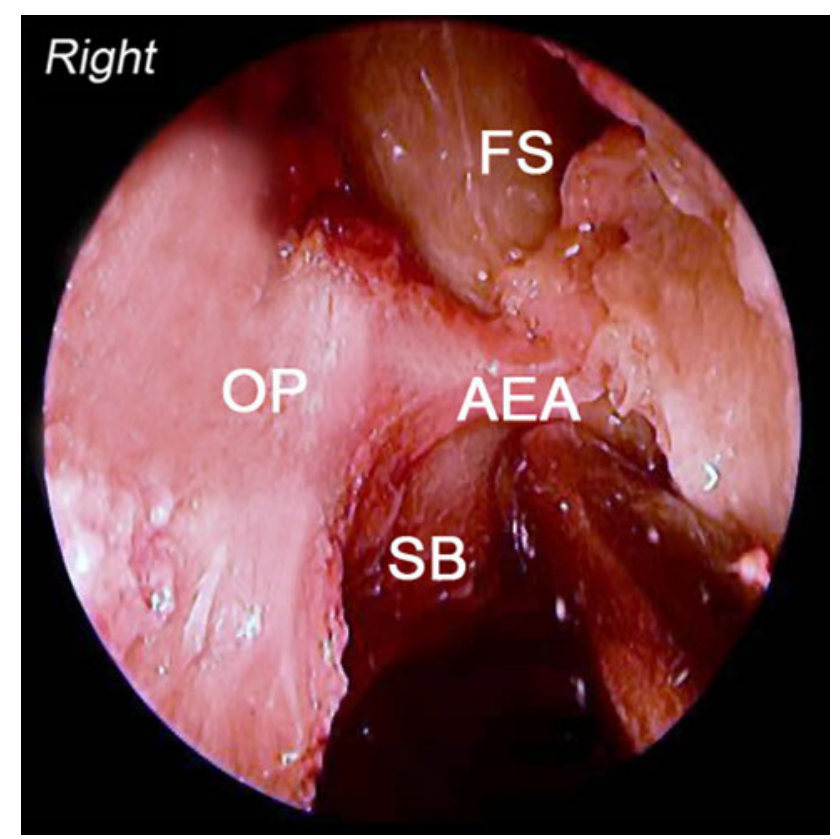

Fig. 1 Right anterior skull base. $O P$ orbital periosteum, $F S$ frontal sinus, $A E A$ anterior ethmoidal artery, $S B$ anterior skull base

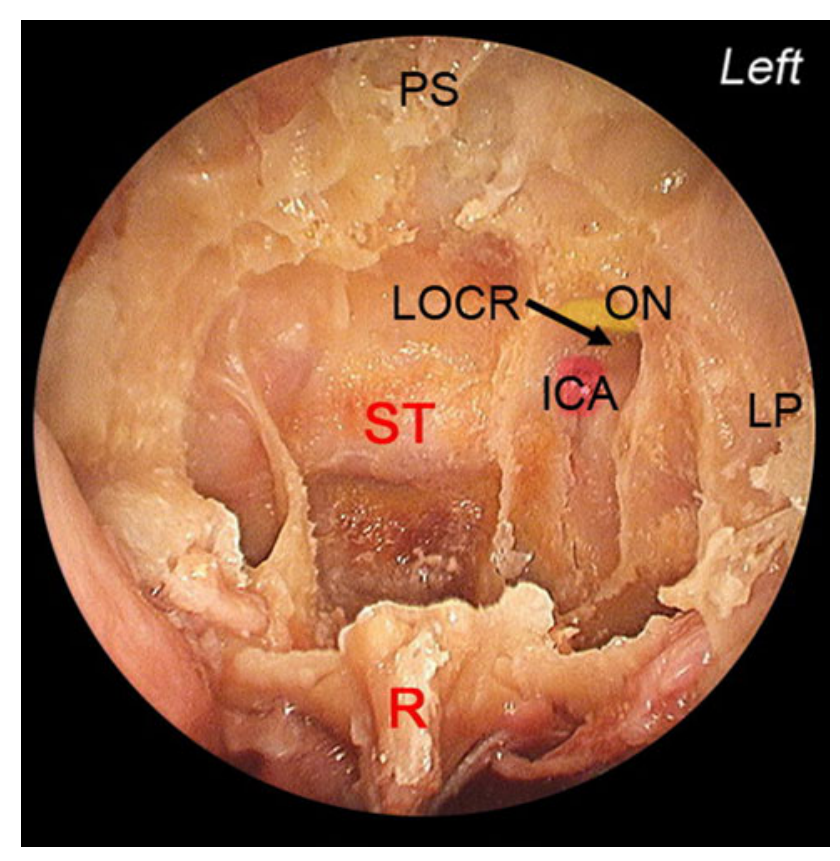

Fig. 2 Superior and lateral wall indentations seen inside the sphenoid sinus. $R$ rostrum of the sphenoid, $S T$ sella turcica, $L P$ lamina papyracea, $P S$ planum sphenoidale, $O N$ optic nerve canal (colored yellow), ICA internal carotid artery indentation (colored red), LOCR lateral optico-carotid recess

inside of the sphenoid sinus with its superior and lateral wall bony indentations namely the carotid protuberance, the optic nerve canal and the lateral optico-carotid recess (LOCR).

\section{Endonasal anatomy}

Orbital apex

Anatomically, the orbital apex (Fig. 3) can be divided into a superior portion consisting of the optic nerve canal and the superior orbital fissure, with its contents, and an inferior portion which includes the inferior orbital fissure, and its contents. Cadaveric dissection begins with a complete exposure of the lamina papyracea and the entire medial orbital wall beginning at the agger nasi cells anteriorly and extending posteriorly till the optic nerve canal in the lateral wall of the sphenoid sinus. Opening of the periorbita is avoided as interference by the orbital fat makes the dissection difficult. The bone around the optic ring at the exit of the optic nerve from orbit is extremely thick. A fine diamond burr is used to decompress the optic nerve at this exit exposing the annulus of Zinn. Further, with the help of a fine curette, lamina papyracea and the bony optic canal are removed, decompressing the optic nerve along its entire course exiting at the orbit and continuing posteriorly till the optic chiasma (Fig. 4).

The LOCR corresponds intracranially to the optic strut which is a bony extension inferior to the anterior clinoid process (Fig. 5). By drilling the optic strut or the LOCR, we join the optic nerve canal with the superior orbital fissure and its contents.

The dissection for the inferior portion of the orbital apex begins with a wide maxillary sinus antrostomy performed using Ostrum's back-biting forceps and identifying the V2 in the roof of the maxillary sinus. Using a fine diamond burr, the V2 canal is decompressed and followed back posteriorly until the foramen rotundum. Figure 6 shows the structures and impressions of landmarks at the orbital apex as seen endoscopically. Using a diamond burr, the bone between V2 and the superior orbital fissure is removed thereby joining the previously joined optic nerve canal and the superior orbital fissure with the inferior orbital fissure. Please note, that endoscopically seen LOCR represents the optic strut in a skull model. In all our dissections we found the optic strut bony landmark to measure less than $10 \mathrm{~mm}$. To avoid injury to the carotid artery, bone removal inferior to the otic nerve should not exceed $1 \mathrm{~cm}$ or the thickness of the optic strut.

\section{Petrous apex}

The vidian nerve canal is the key landmark in the endoscopic approach of the petrous apex (see Fig. 7). Dissection of the vidian canal courses posteriorly towards the anterior genu of the petrous carotid artery. As we drill the floor of the spenoid sinus, vidian canal is identified at the junction of the medial pterygoid plate and the lateral sphenoid floor. 
Fig. 3 Schematic representation of the right orbital apex

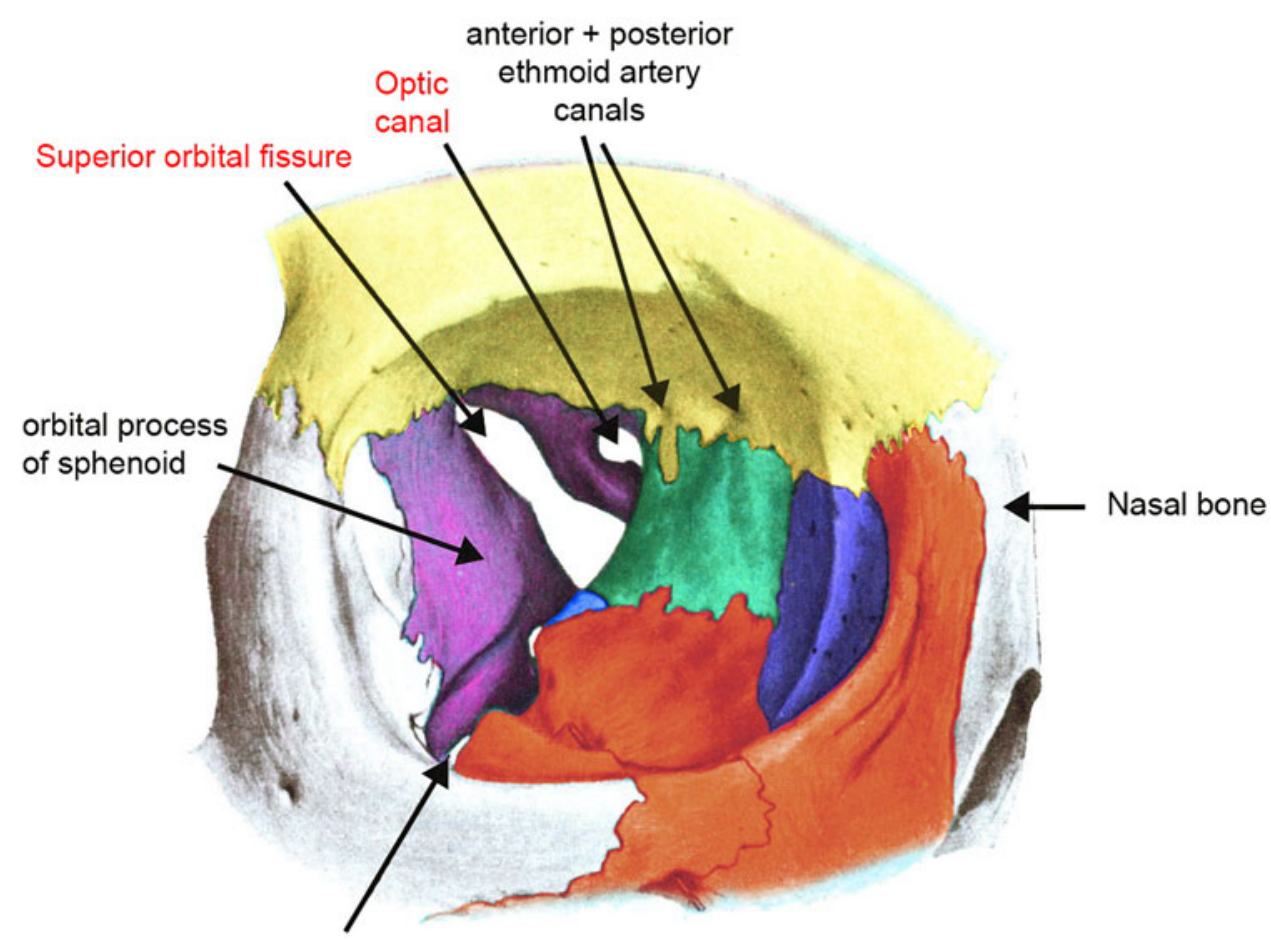

Inferior orbital fissure

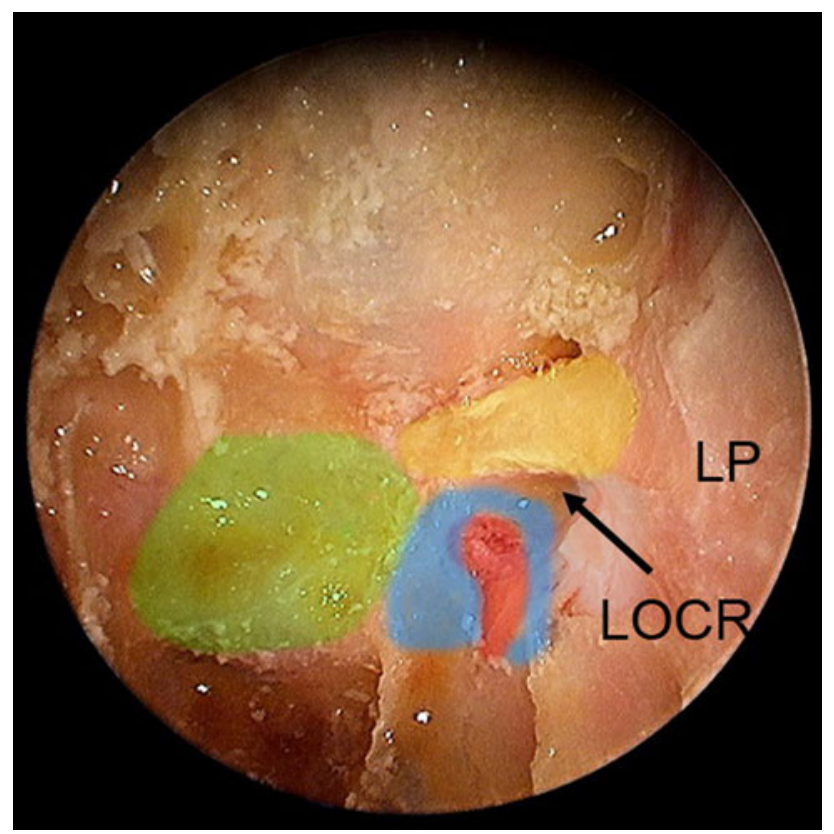

Fig. 4 Left lateral wall of the sphenoid sinus. Various colors have been used to show structural impressions-green sella, red carotid bulge, blue cavernous sinus, yellow optic nerve canal. LP lamina payracea, LOCR lateral optico-carotid recess

Using a small diamond burr, bone removal continues along the inferomedial border of the vidian canal. At this time, the internal carotid artery (ICA) is located along it superior border (Fig. 8). By drilling inferiorly, the exact position of the ICA can be established and the petrous apex exposed,

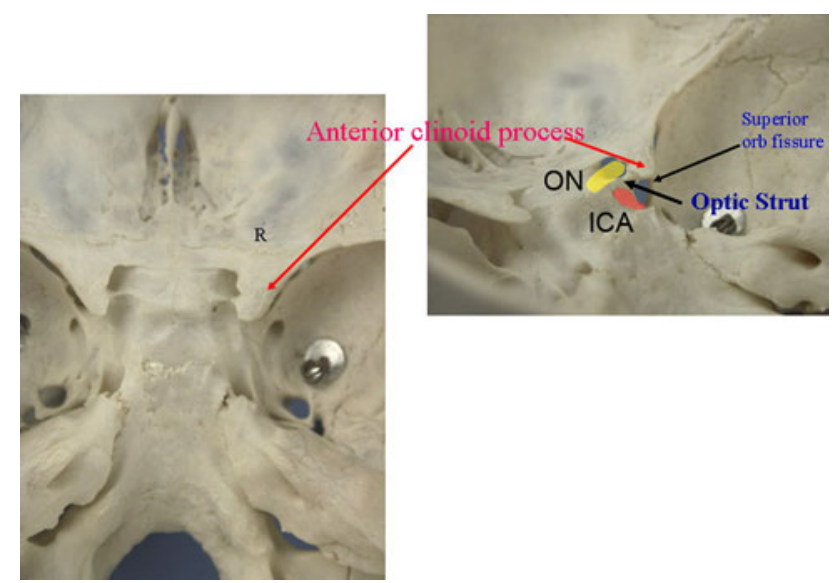

Fig. 5 Plastinated skull model showing the right optic strut. $O N$ optic nerve (yellow), ICA internal carotid artery (red)

keeping the ICA always superolateral to the vidian canal. In all our 12 dissected cadaveric sides the ICA was never located inferior and medial to the vidian canal. Figure 9 shows the petrous carotid artery with the upper cranial nerves traversing through the cavernous sinus (which has been removed in the figure).

Pterygopalatine fossa

A wide anterior maxillary antrostomy and inferior turbinectomy is performed to allow a transantral-transturbinal approach to the pterygopalatine fossa (PPF) (Fig. 10). 


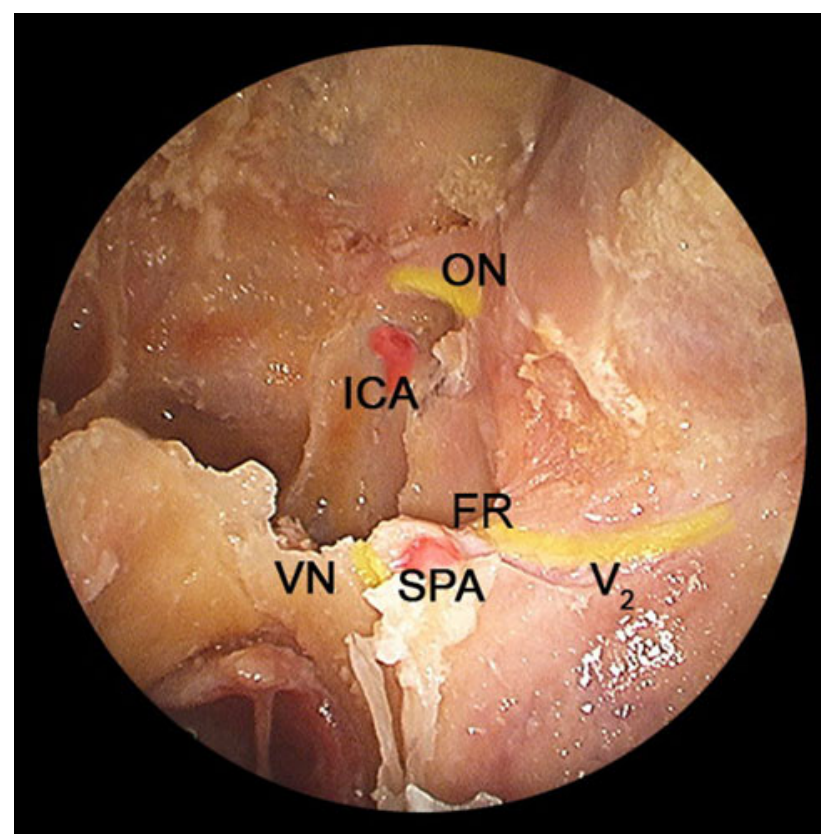

Fig. 6 Structures and landmark impressions at the left orbital apex. $O N$ optic nerve (yellow), ICA internal carotid artery indentation (red), $V N$ vidian nerve (yellow), SPA sphenopalatine artery (red), FR foramen rotundum, $V 2$ maxillary nerve (yellow)

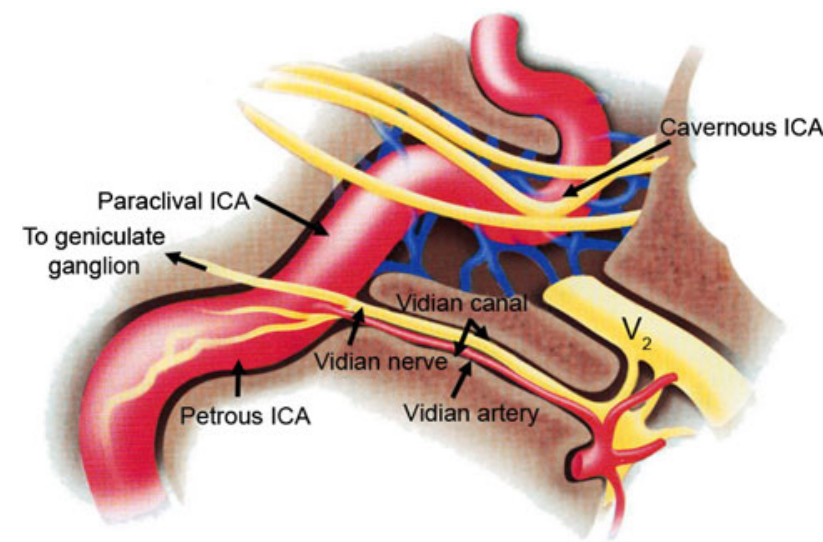

Fig. 7 Schematic representation showing the right vidian canal consisting of the vidian artery and nerve leading to the petrous carotid artery

Nasal mucosa just anterior to the posterior attachment of the middle turbinate is elevated to visualise the ethmoidal crest, which is removed and the sphenopalatine artery identified. Posterior wall of the maxillary sinus is quite thin and easily removed using rongeurs. Bechat's fat pad is dissected to identify the contents of the PPF. Following the sphenopalatine artery laterally, third part of the internal maxillary artery and its branches are traced (Fig. 11). The pterygopalatine ganglion (PPG) is identified as an oval shaped pale yellow coloured structure situated posterior to the arterial arcade. The PPG recieves afferents from the vidian, V2, and the posterior palatine nerves. The dissection

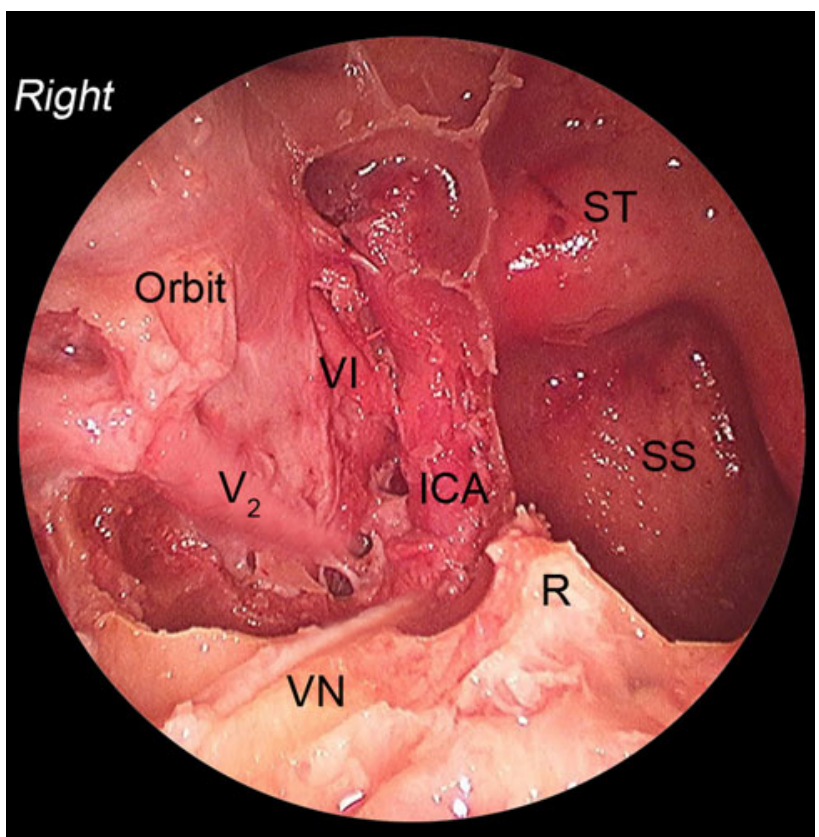

Fig. 8 Right petrous apex. ICA internal carotid artery, $V N$ vidian nerve, $S S$ sphenoid sinus, $S T$ sella, $R$ rostrum, $V I$ abducens nerve, $V 2$ maxillary nerve

continues in a medial to lateral direction, identifying branches of the internal maxillary artery anterior to the pterygoid muscles. Tracing the V2 posteriorly and identification of the foramen rotundum limits the posterior dissection. Anatomically, the PPF communicates with the infratemporal area (ITF) through the pterygomaxillary fissure. Endoscopically (Fig. 12), the area medial to V2 is the PPF, and the area lateral to the $\mathrm{V} 2$ is the infratemporal fossa $[2,5]$. Not many veins are encountered in the PPF. Posteriorly, the V2 is traced until the foramen rotundum, and lateral dissection is continued till the temporalis musle. It is difficult to dissect and identify V3 in the abundant fat that occupies the PPF.

\section{Discussion}

There are extensive descriptions in literature of open approaches to midline skull base lesions. However, these open procedures are extremely complex, traverse through major neuro-vascular structures requiring sometimes retraction of the brain with subsequent morbidity $[5,6]$. Recently transnasal endoscopic approaches have been reported for the resection of intra and extra-cranial lesions involving the midline skull base [1]. This has opened surgical corridors for the otorhinolaryngologist, skilled in endoscopic surgery, to perform interventions in these areas in collaboration with opthalmologists and neurosurgeons. The initial step in the transnasal endoscopic skull base dissection 


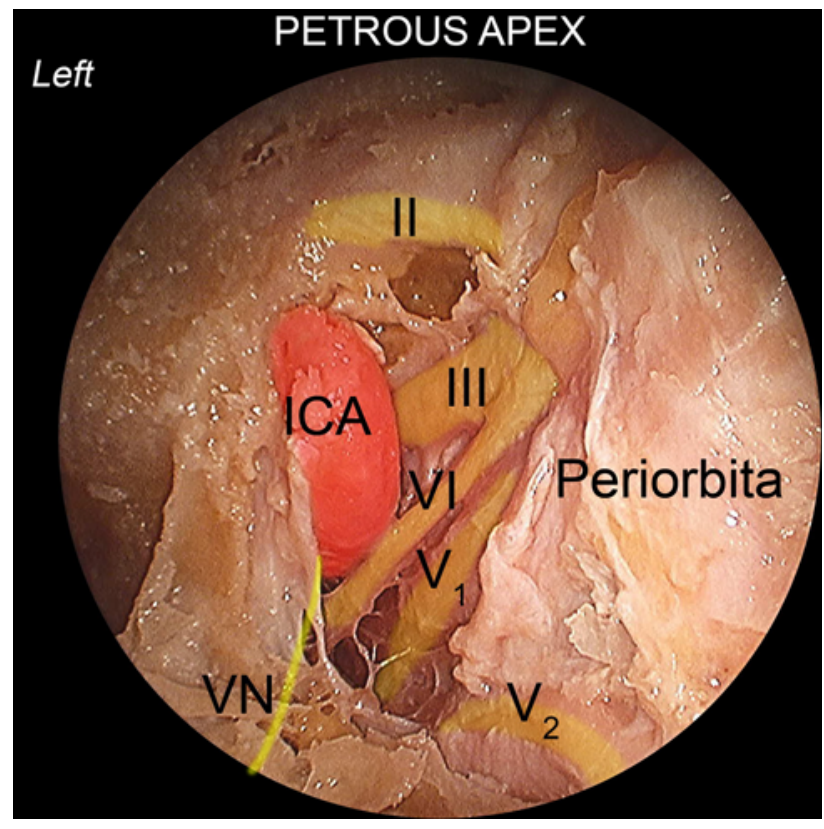

Fig. 9 Left petrous apex. Cavernous sinus has been removed to show the transcavernous course of upper cranial nerves (nerves colored in yellow and artery in red). ICA internal carotid artery, $V N$ vidian nerve

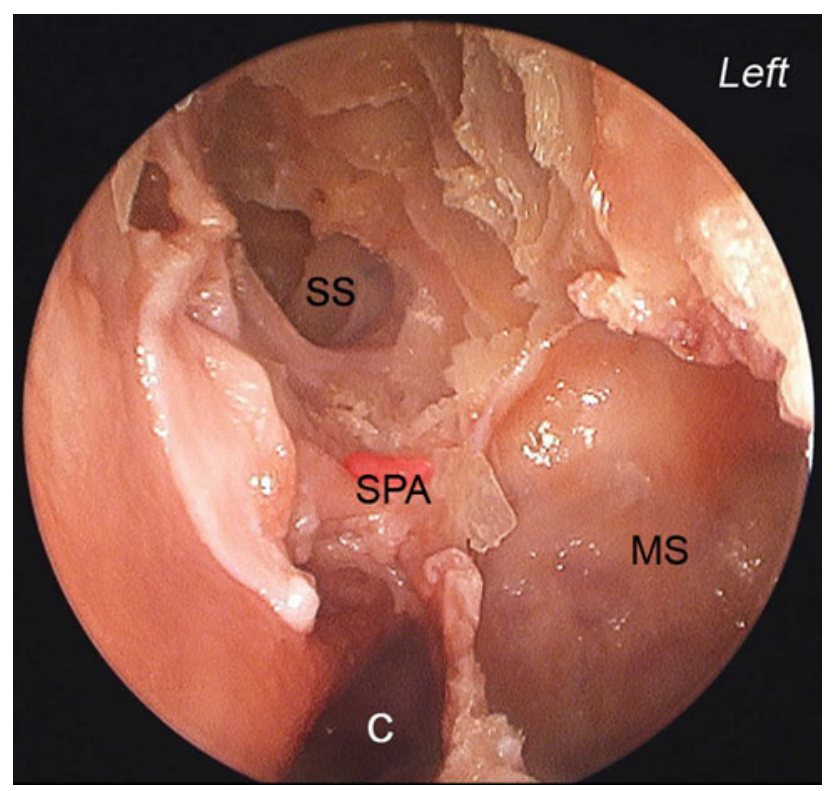

Fig. $10 M S$ the left maxillary sinus, $S S$ sphenoid sinus, $S P A$ sphenopalatine artery $(r e d)$ and $C$ posterior choana

begins with ethmo-sphenoidectomy and a wide maxillary antrostomy. A posterior septectomy and right middle turbinectomy allows a bi-nostril 4 hand dissection for better instrumentation and surgical collaboration. The chief and assistant surgeon can use both their hands, while accessing the skull base through both nostrils.

For dissecting the orbital apex we find following landmarks to be extremely useful-the optic nerve, LOCR, bulge of the ICA, optic strut and V2. The distance between

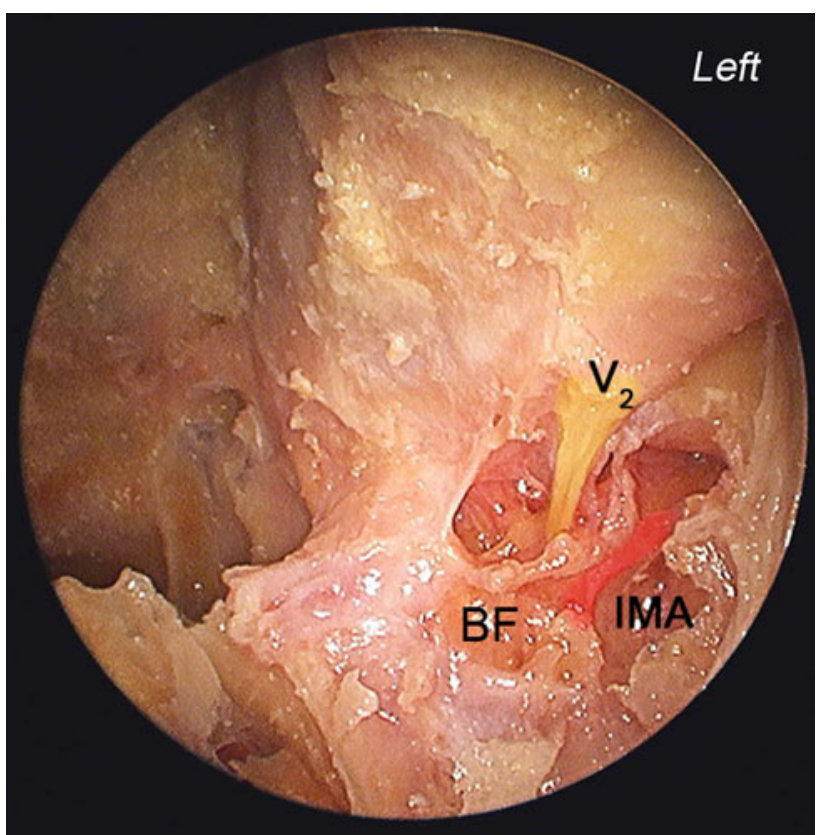

Fig. 11 Left pterygopalatine fossa (PPF). IMA internal maxillary artery (red), V2 maxillary nerve (yellow), BF Bechat's fat pad

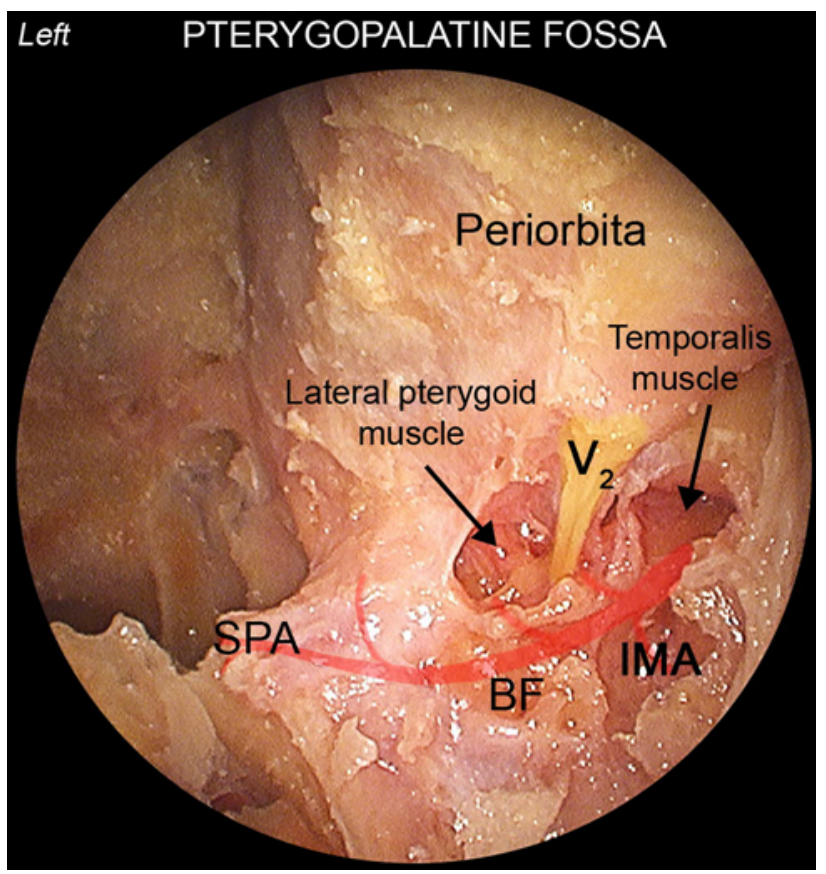

Fig. 12 Left PPF communicating with the infratemporal fossa. SPA sphenopalatine artery (red), IMA internal maxillary artery (red), V2 maxillary nerve (yellow), $B F$ Bechat's fat pad

the ICA and the optic nerve measures $2-10 \mathrm{~mm}$ and corresponds to the LOCR or the optic strut [2]. The LOCR seen during endoscopy is the optic strut seen intracranially.

The petrous apex is bounded by the bony labyrinth and ICA anteriorly, the posterior cranial fossa and Dorello's canal (cranial nerve VI) posteriorly, the middle cranial 
fossa and Meckel's cave superiorly, and the jugular bulb and inferior petrosal sinus inferiorly [7]. The ICA is of critical importance for endoscopic endonasal approaches to the petrous apex [8]. One of the key anatomical relationships that must be understood to achieve endoscopic control of the petrous carotid artery, is the relationship of the vidian canal to the anterior genu of the ICA and the surrounding foramina and fossae [6]. The vidian canal runs from the pterygopalatine fossa to the foramen lacerum and houses the vidian nerve and in $30 \%$ of cases the vidian artery is a direct branch of the ICA [7]. The vidian nerve and the V2 represent reliable landmarks for dissecting the petrous apex area. The vidian nerve canal is identified at the junction of the medial pterygoid plate and the lateral sphenoid sinus floor. Dissection can be continued posteriorly but remaining inferomedial to the vidian nerve, and the ICA lies lateral and superior to the nerve. The vidian nerve, V2 and a tangential line drawn between them form a triangle and the ICA lies at the apex of this triangle. In all our 12 dissected sides, we have never seen the ICA being medial or inferior to the vidian canal, thus defining the areas which can be safely removed without injuring the carotid artery.

The PPF is a pyramidal space located between the pterygoid bone posteriorly, the palatine bone anteromedially, and the maxillary bone anterolaterally. The base of the medial and lateral pterygoid processes forms its posterior surface, where the anterior opening of the vidian canal is located medially and the foramen rotundum is located laterally. Inferiorly, the pterygoid processes fuse with the pyramidal process of the palatine bone. The vertical plate of the palatine bone articulates with the medial surface of the medial pterygoid plate posteriorly and with the medial wall of the maxillary sinus anteriorly. In the upper part, the anterior part of the perpendicular plate of the palatine bone ends in the orbital process anteriorly and the sphenoid process posteriorly. The orbital process attaches to the orbital surface of the maxillary bone and to the inferior surface of the sphenoid bone. The sphenoid process attaches to the base of the medial pterygoid plate. These two processes, when articulated, form the sphenopalatine foramen, through which the sphenopalatine nerve and artery pass. The external part of the posterior wall of the maxillary sinus constitutes the anterolateral surface of the pterygopalatine fossa [2]. The PPF communicates laterally with the infratemporal fossa through the pterygomaxillary fissure, with the orbit anteriorly through the inferior orbital fissure, with the middle cranial fossa posterolaterally through the foramen rotundum, with the foramen lacerum posteromedially through the vidian canal, with the nasopharynx medially through the palatovaginal canal, and with the oral cavity inferomedially through the greater and lesser palatine foramina [9]. The anterior compartment of the pterygopalatine fossa contains the third segment of maxillary artery and
Table 1 Anatomic area with its key landmarks

\begin{tabular}{ll}
\hline Dissection area & Landmarks \\
\hline Orbital apex & Optic nerve; \\
& Lateral optico-carotid recess; \\
& Carotid artery bulge; \\
& Optic strut; \\
& V2 \\
& Vidian canal (vidian nerve and artery); \\
& V2-foramen rotundum \\
Petrous apex & Sphenopalatine foramen and artery; \\
Pterygopalatine fossa & V2 \\
\hline
\end{tabular}

its branches and the pterygoid muscles [10]. Identification of the V2 and its posterior dissection until the foramen rotundum marks the posterior limit of the PPF dissection. The posterior compartment of the fossa contains the pterygopalatine ganglion and its branches and the maxillary nerve. The close relationship between the pterygopalatine fossa and several anatomic regions-intracranial and extracranial, facilitates spread of tumours and infections from the head and neck to the cranial base. Many vascular tumors (angiofibroma), neural tumors (schwannoma) arise in the PPF leading to infratemporal, orbital and intracranial extensions. For this reason, the pterygopalatine fossa, despite its small size, assumes a particular surgical interest in the cranial base neurosurgery. Table 1 summarises endoscopic landmarks for the orbital apex, the petrous apex and the PPF.

Neoplasms that most commonly occupy the orbital apex and can be treated by the transnasal endoscopic approach (TEA) include cavernous hemangioma, schwannoma, neurinoma and fibrous dysplasia $[2,3,7]$. The orbital apex is an enclosed space containing several important neural and vascular structures, including the optic nerve, vascular supply to the eye and orbit, and cranial nerves responsible for ocular movement. In addition, it is located adjacent to the ICA and cavernous sinus. Vision can be compromised when the orbital apex is narrowed by adjacent tumor or bony overgrowth. Transnasal endoscopic removal of the medial bony wall of the orbital apex and incising the periorbita can reverse compressive optic neuropathy $[11,12]$.

Petrous apex lesions may remain undetected for extended periods because patients often complain of vague or indistinct symptoms that delay diagnosis. Headaches, atypical facial pain, mixed hearing loss, vertigo, eustachian tube dysfunction, and middle ear effusion may be the initial manifestations of an extensive petrous apex lesion. The TEA has been effectively used in treating expansile benign petrous apex lesions like cholesteatoma, cholesterol granuloma and primary mucocele $[2,7]$. 
Certain PPF lesions like angiofibroma, schwannoma, neuroma are ideal to be treated by the TEA [1,2]. This approach is minimally invasive and better suited for surgical debridement of fungal infections (aspergillosis, mucormycosis), than the open transfacial approaches. Even though this region is relatively small and its location is deep, in the presence of tumors, especially benign ones, all the neurovascular structures are displaced by the lesion itself, which creates or enlarges the surgical corridors. Under these conditions, endoscopically it is relatively easy to manipulate the blood vessels and nerves and then excise these lesions.

Malignant tumors (primary/recurrent/metastatic epidermoid, adenoid cystic) at the orbital apex, petrous apex and PPF can be treated by transnasal endoscopic debulking followed by radiotherapy, and this has been accepted as an effective means of surgical palliation [2], though results and surgical aims are debatable. Complications of the expanded endonasal approach are the same as open approaches: neural and vascular injury, meningitis and CSF leak. The main problem faced during surgery is bleeding, and only an experienced team can effectively use the bipolar electrocautery or manage cavernous sinus bleeding with precise focal packing without increasing the neurovascular injury. Another important factor in consideration is the reconstruction of ventral skull base defects after endoscopic excisions. Multilayered closures, vascularised nasoseptal mucosal flaps, balloon stenting and surgical bioglues have been sentinel events in reconstruction that have been effective in closing these defects reducing chances of CSF leaks [10].

At present, these surgical resection techniques are being practised at very few centers and long term results are awaited. The most critical issue to be addressed is avoiding trauma to the carotid artery and the optic nerve. One must have a thorough knowledge of various radiological investigations like the CT, MRI and 3D reconstructional images of the skull base. Periodic discussions with radiological collegues with special interests in the skull base betters our understanding of this complex anatomy. Intraoperatively, use of navigational techniques and a systematic use of the doppler have been very useful in our limited experience. This approach adds choice, variety and versatility to the armamentarium of a skull base surgeon and may serve as a good alternative to transfacial approaches which are complex and more morbid.

\section{Conclusions}

Surgical interventions in complex areas of orbital apex, petrous apex and the pterygopalatine fossa is a long learn- ing curve and begins in the dissection rooms. One must realise that anatomy is a science of recognition and not of discovery, hence surgeons need to have adequate knowledge of endoscopic nasal anatomy before embarking on treating lesions of these complex regions. Each of these areas can be approached systematically, by defining and identifying reliable landmarks. These approaches could be effectively used for certain selected lesions of the anterior skull base, which otherwise would require more complex and morbid procedures. We intend to plastinate our dissected specimens for teaching purposes. Obviously, these approaches require collaboration between otorhinolaryngologists and neurosurgeons.

Conflict of interest None.

\section{References}

1. Cavallo LM, Messina A, Cappabianca P, Esposito F, De Devitiis E, Gardner P, Tschabitcher M (2007) Extended endoscopic endonasal transsphenoidal approach to the suprasellar area: anatomic considerations-part 1 . Neurosurgery 61:24-34

2. Anand VK, Schwartz TH (2007) Practical endoscopic skull base surgery. Plural Publishing, San Diego

3. Jho HD, Ha HG (2004) Endoscopic endonasal skull base surgery: part 1-the midline anterior fossa skull base. Minim Invasive Neurosurg 47:1-8

4. Cavallo LM, Mesina A, Cappabianca P, Gardner P, Tschabitscher M (2005) Endoscopic endonasal surgery of the midline skull base: anatomical study and clinical considerations. Neurosurg Focus 19(1):E2

5. Malgro F, Solari D, Cavallo LM, Samii A, Cappabianca P, Paterno V (2006) Endoscopic endonasal approach to the lateral recess of the sphenoid sinus via the pterygopalatine fossa: comparison of endoscopic and radiological landmarks. Neurosurgery 59(ONS Suppl 4):237-243

6. Kassam AB, Gardner P, Snyderman C, Carrau R (2008) Expanded endonasal approach: vidian canal as a landmark to the petrous internal carotid artery. J Neurosurg 108:177-183

7. Kassam AB, Gardner P, Snyderman C et al. (2005) Expanded endonasal approach: fully endoscopic, completely transnasal approach to the middle third of the clivus, petrous bone, middle cranial fossa, and infratemporal fossa. Neurosurg Focus 19:E6, $1-10$

8. Brackmann DE, Toh EH (2003) Surgical management of petrous apex cholesterol granulomas. Otol Neurotol 23:529-533

9. Osborn AG (1980) The vidian artery: normal and pathologic anatomy. Radiology 136:373-378

10. Snyderman CH, Kasam AB, Carrau R, Mintz A (2007) Endoscopic reconstruction of cranial base defects following endonasal skull base surgery. Skull base: an interdisciplinary approach 17(1):75-76

11. Yeh S, Foroozan R (2004) Orbital apex syndrome. Curr Opin Ophthalmol 15(6):490-498

12. Schick U, Dott U, Hassler W (2003) Surgical treatment of orbital cavernomas. Surg Neurol 60(3):234-244 